ORIGINAL ARTICLE

\title{
Prevalence of HIV Associated Neurocognitive Deficit among HIV Positive People in Ethiopia: A Cross Sectional Study at Ayder Referral Hospital
}

\author{
Tilahun Belete $^{1^{*}}$, Girmaw Medfu $^{2}$, Ephrem Yemiyamrew $^{3}$
}

OPEN ACCESS

Citation: Tilahun Belete, Girmaw Medfu, Ephrem Yemiyamrew. Prevalence of HIV Associated Neurocognitive Deficit among HIV Positive People in Ethiopia: A Cross Sectional Study at Ayder Referral Hospital. Ethiop J Health Sci 2017;27(1):67-76. doi: http://dx.doi.org/10.4314/ejhs.v27i1.9

Received: October 12, 2016

Accepted: October 16, 2016

Published: January 1, 2017

Copyright: Tilahun B, et al. This is an open access article distributed under the terms of the Creative Commons

Attribution License, which permits unrestricted use, distribution, and reproduction in any medium, provided the original author and source are credited. Funding: Nil

Competing Interests: The authors declare that this manuscript was approved by all authors in its form and that no competing interest exists.

Affiliation and Correspondence: ${ }^{1}$ Psychiatry Unit, School of Nursing, College of Health Sciences, Mekelle University, Ethiopia

"Email: tilahunbe100@gmail.com

\section{ABSTRACT}

BACKGROUND: HIV associated neurocognitive deficit impairs motor activity, neuropsychiatric functioning, daily activity and work activity usually due to the immune suppression effect of the virus. Sub-Saharan region including Ethiopia is the region with the highest burden of HIV. However, a few studies are found on this aspect nationally. This study was aimed at determining the prevalence and the factors associated with cognitive impairment among HIV positive people in Ethiopia who attended Ayder Comprehensive Specialized Hospital.

METHOD: A hospital based cross sectional study was employed on 234 participants selected using systematic random sampling technique. Data was collected thrpugh face-to-face interview, observation and document review. International HIV dementia scale, activity of daily living scale and Hospital Anxiety and Depression scale were used to assess neuro cognitive deficit, activity of daily living, anxiety and depression respectively. The data was analyzed by using SPSS window 20.

RESULT: About $88 \%$ of the subjects were receiving highly active antiretroviral therapy. The magnitude of Neuro cognitive deficit was $33.3 \%$ (95\% CI; $27.7 \%$ - 40.6\%). Impairment in the activity of daily living was observed on $9.8 \%$ of the participants. Besides, $55.6 \%$ and $67.1 \%$ had anxiety and depressive disorders respectively. Late clinical stage of the illness $(A O R=4.2(95 \% \mathrm{CI}$; $1.19,14.44)$ ) and impairment in the activity of daily living were significantly associated with neurocognitive deficit (AOR= 7.19 (95\% CI; 1.73, 21.83).

CONCLUSION: A higher prevalence of neurocognitive deficit was observed that was related to impaired activity of daily living and being in late stages of the illness. Hence, this should be a strong alarm for early detection of the problem and consistent review of the treatment regimen.

KEYWORDS: HIV associated Neurocognitive Deficit, Neuro cognitive deficit, HIV Associated Dementia, Cognitive Impairment, International HIV Dementia Scale, Ethiopia 


\section{INTRODUCTION}

The area with the highest burden of Human Immune Deficiency Virus (HIV) that accounts for $75 \%$ of the case is Sub-Saharan region. This is the region with only $12 \%$ of the global population (1). Neurocognitive deficit (NCD) occurs mainly due to HIV among HIV positive people $(2,3)$. The problem in subSaharan area is expected to be large as HIV infection and its sequel are prevalent in the region $(2,4)$. Previous studies conclude that neurocognitive deficit has been found at any stage of the illness $(5,6,7,8)$.

Neurocognitive deficit is a deficit in neurological activities, motor activities, psychological functioning, daily activities and activities at work place. The major cause of morbidity and disability among HIV positive people has been accounted to $\mathrm{NCD}$, although the problem is rarely studied in the region. Deficits in different areas of life such as cognitive, motor, emotional and even personality changes can be caused by HIV especially lately as the disease progression fastens. These problems range from the asymptomatic impairments to mild forms and to the more severe HIV dementia $(3,4,9,10,11)$.

A Metaanalysis (systematic review) found out that in sub-Saharan region, a higher prevalence was observed in the pre-highly active anterotroviral therapy (HAART) participants in which neurocognitive impairment (NCI) preHAART was $42.37 \%$, and among those on HAART was $30.39 \%$ (2). Respective NCI estimates in studies from Uganda were $46.49 \%$. In addition, NCI was more common among patients with a concomitant psychiatric ailment. And, the study concluded that HIV strongly predisposes to NCI leading to a huge burden in Sub-Saharan Africa (4).

The more common impairments are the asymptomatic and mild forms that are more prevalent with advanced age (12). The problem of NCD in sub-Saharan area ranges from 20\% - 37\% as reported in prior studies $(2,11,13)$. A report in the multi-center international studies that were conducted in Thailand, Zaire, Kenya, Brazil and Germany revealed that the neuropsychological deficit among HIV-positive people ranged from $13 \%$ in Brazil to $19 \%$ in Zaire $(14,15,16)$.

A study conducted in Northern Nigeria in 2014 in which a total of 418 patients participated showed that a total of $21.5 \%$ participants were found to have cognitive impairment. Using a Frascati criterion, $13.3 \%$ were found to have HIV associated dementia (HAD), $42.2 \%$ had mild neurocognitive deficit, and $44.4 \%$ had asymptomatic neurocognitive impairment, within this group. Cognitive impairment was significantly associated with duration of illness, CD4 count, viral load, education, severity of the illness and history of complete stopping of HAART in the past (17).

A similar study from India between October 2011 and September 2012, on 80 HIV-positive individuals randomly selected from the ART Centre, the patients were clinically examined and tested using International HIV dementia scale (IHDS). Accordingly, all HIV-positive participants who were already on HAART were found to have neurocognitive deficit; the overall prevalence was $32.5 \%$ (18).

In a similar finding. all participants were either in World Health Organization (WHO) clinical stage I or stage II of HIV illness and $87.1 \%$ of them were on HAART. The prevalence of HIV associated neurocognitive deficit (HAND) was $90 \%$. And it was determined by IHDS (19).

In Malawi and Botswana, studies conducted on 106 and 120 patients reported that in the former symptomatic neurocognitive impairment was present in 15\%; 55\% fulfilled Frascati criteria for asymptomatic neurocognitive impairment (20). As well, 38\% had severe form of NCD (HIV associated dementia) in Botswana that was significantly associated with educational level and age. In that study, $24 \%$ of the subjects also had co- existing depression (21). However, the level of education significantly affected performance on all three cognitive measures, and age affected processing speed and performance on the IHDS. Depression and current CD4 count did not affect performance on any of the cognitive measures (21).

A study conducted in Ethiopia in 2014 on 423 HIV positive participants by using IHDS, determined that the prevalence of HIV associated

DOI: http://dx.doi.org/10.4314/ejhs.v27i1.9 
dementia (severe form of neurocognitive impairment) was $24.8 \%$. Also, low levels of education, older age of 50 years or above, co morbid opportunistic infection and substance use were significantly associated with HIV dementia (22).

Neurocognitive impairment is one of the serious problems among HIV-positive people. It can lead to morbidity, treatment non- adherence, faster progression of the illness, disability and bed ridden state if we fail to detect it early. Even it can result in death in severe cases despite easier methods to screen the problem.

It is important to study such problems in order to improve the well being and quality of life of HIV-positive people. Nevertheless, there is scarcity of studies on this research title in Ethiopia Therefore, the findings of this study might be valuable to different stakeholders, healthcare facilities and clinicians who are working in the scope. Thus, this study aimed at assessing the prevalence and factors associated with cognitive impairment among HIV-positive people attending at Ayder Comprehensive Specialized Hospital (ACSH), Mekelle, Ethiopia.

\section{METHODS AND SUBJECTS}

Mekelle is the capital city of Tigray Administrative Regional State located $783 \mathrm{kms}$ North of Addis Ababa. The city is divided into seven sub-administrative units, and it has one comprehensive specialized hospital (Ayder), two other public hospitals and one military hospital. A hospital based cross sectional study was conducted at Ayder Comprehensive Specialized Hospital between February and June 2016 (23).

All HIV positive people who had follow up at the hospital were the source population. And, the study population included those participants who visited the hospital during the data collection period.

The inclusion criteria was age between 18-64 years (because age related neurocognitive deficit i.e. dementia starts from age of 65 years) and having documented HIV-positive status. Those with known cognitive impairment unrelated to HIV (neurological, severe medical illnesses unrelated to HIV, severe mental illness) were excluded from the study.

Sample size and sampling procedure: The actual sample size was determined by using the single population proportion formula where the following were considered: $95 \%$ confidence interval, $24.8 \%$ proportion of neurocognitive deficit among HIV positive patients (22) and 5\% margin of error. Since the total population was 1184, we e employed correction formula, and then added $10 \%$ estimated non-response rate that made a final sample size of 254 .

The participants were selected through systematic random sampling technique after having the monthly client flow to the hospital. Two psychiatric nurses were employed to collect data. Data were collected through face-to-face interview, observation and document review. Local language versions of International HIV dementia scale, activity of daily living scale and hospital anxiety and depression scale were used to assess neurocognitive deficit, activity of daily living, anxiety and depression respectively. These tools have been validated in sub-Saharan region, and HADS has been validated in Ethiopia, too, $(24,25,26,27)$.

Translation of the tools was carried out from their English version into local language version (Tigrigna) by professional translators, and the local language version was translated back into English by another professional with the help of a mental health specialist. Then, consistency was assured. Also, training was provided to the data collectors for one day on what information to gather and how to approach each client. A pretest was done at another public facility on $5 \%$ of the sample to check for necessary modifications. Also, daily supervision was employed during the data collection period. Daily data checkup and entry were also done.

\section{Operational definitions}

- HIV Associated Neurocognitive Deficit: It is a reduction or impairment of brain function; any patient who score less than or equal to 9.5 from IHD scale (24) has this problem.

- Activities of daily living: A score of 4 or less on Ketz Activity of Daily Living scale is considered to be a person impaired in his/her activity of daily living $(25,26)$. 
- Anxiety: A score of 8 or more on anxiety symptoms on Hospital Anxiety and Depression scale is considered that the person has anxiety (27).

- Depression: A score of 8 or more of depression symptoms on Hospital Anxiety and Depression scale is considered that the person has depression (27).

Data checkup, coding, entry and analysis were computed using SPSS window 20. To determine the degree of association between different variables and neurocognitive deficit, logistic regression was employed. Variables with p-value of 0.2 or less in the bivariete analysis were selected for multivariate analysis. The degree of association was determined with adjusted odds ratio (and 95\% confidence interval).

Ethical consideration: Ethical was obtained from Health Research Ethics Review Committee of the College of Health Sciences, Mekelle University. Permission was obtained from the hospital, and then consent was collected from the study participants before filling each questionnaire respecting their rights to withdraw from the study at any time. Confidentiality was assured for all the information provided, and no personal identifiers were used on the questionnaire. Participants who had neurocognitive deficit were resent to the clinicians for further evaluation.

\section{RESULTS}

A total of 234 HIV-positive people who had follow-up at Ayder Comprehensive Specialized Hospital participated in the study. Thus, the response rate was $92.1 \%$.

Socio-demographic characteristics: The respondents were predominantly females $151(64.5 \%)$. The mean age (standard deviation) of participants was $38.26( \pm 9.94)$ with a range of 18 to 64 years. The proportions of participants in the age group of 18 to 30 years, 31 to 40 years and above 40 years were $32.1 \% 34.1 \%$ and $33.8 \%$ respectively.

Table 1: Socio demographic characteristics of HIV-positive people, Ayder Comprehensive Specialized Hospital, Ethiopia, June 2016 ( $\mathrm{n}=234)$.

\begin{tabular}{llll}
\hline Characteristics & & Frequency & Percent \\
\hline Sex & Female & 151 & 64.5 \\
Age (years) & Male & 83 & 35.5 \\
& $18-30$ & 75 & 32 \\
Marital status & $31-40$ & 80 & 34.2 \\
& $>40$ & 79 & 33.8 \\
& Single & 40 & 17.1 \\
& Married & 135 & 57.7 \\
& Divorced & 33 & 14.1 \\
Educational status & Widowed & 26 & 11.1 \\
& No education & 110 & 47 \\
& Primary & 61 & 26.1 \\
& Secondary & 30 & 12.8 \\
Occupation & Higher education & 33 & 14.1 \\
& Laborer & 48 & 20.5 \\
& Civil servant & 53 & 22.6 \\
& Merchant & 64 & 27.4 \\
& Housewife & 50 & 21.1 \\
& Farmer & 16 & 6.8 \\
& Others & 3 & 1.3 \\
Monthly income (Birr) & $<500$ & 123 & 52.6 \\
& $501-1000$ & 32 & 13.7 \\
& $1001-1500$ & 50 & 21.1 \\
& $>1500$ & 29 & 12.4 \\
& & &
\end{tabular}

DOI: http://dx.doi.org/10.4314/ejhs.v27i1.9 
Educational status showed that $69(29.5 \%)$ of them were not able to read and write. Additionally, $53(22.6 \%)$ and $64(27.4 \%)$ were daily laborers and government employee by occupation respectively. Married participants were 135(57.7\%). Also, $206(88 \%)$ and $169(72.2 \%)$ were Tigray by ethnicity and Orthodox Christian by religion respectively; $123(52.6 \%)$ of the respondents had a monthly income of 500 Birr or less (Table 1).

Among 234 participants, 206(88\%) were receiving HAART; about $54.3 \%$ were on follow- up for 6 or more years. Also, 139(59.4\%) patients were at stage 1 while $64(27.4 \%)$ were at stage 2 according to WHO clinical staging. In addition, the recent CD4 count (within 3 months of the data collection period) was 351-500 cells/dl and 201350 cells/dl for about $32.9 \%$ and $31.2 \%$ of the subjects respectively. Opportunistic infections were observed in $14.5 \%$ of the participants. Besides, current use of psycho-active substances was reported in $13.7 \%$ of them (Table 2).

Table 2: Clinical factors of HIV Positive People, Ayder Comprehensive Specialized Hospital, Ethiopia, June $2016(n=234)$.

\begin{tabular}{llll}
\hline Clinical factors & & Number & $\%$ \\
\hline CD4 count (cells/dl) & $<201$ & 25 & 10.7 \\
& $201-350$ & 73 & 31.2 \\
& $351-500$ & 77 & 32.9 \\
Duration of illness & $>500$ & 59 & 25.2 \\
(Years) & 1 or less & 29 & 12.4 \\
& $2-4$ & 78 & 33.3 \\
Clinical stage (WHO) & 5 or more & 127 & 54.3 \\
& Stage I & 139 & 59.4 \\
& Stage II & 64 & 27.4 \\
Status of HAART & Stage III & 25 & 10.7 \\
& Stage IV & 6 & 2.6 \\
Current OIs & On HAART & 206 & 88 \\
& Pre-HAART & 28 & 12 \\
& No & 200 & 85.5 \\
& Tuberculosis & 17 & 7.4 \\
& Pneumonia & 3 & 1.3 \\
\hline
\end{tabular}

Magnitude of NCD: Of the study participants, 78 scored 9.5 or less on IHDS. Thus, the magnitude of HIV associated neurocognitive deficit was $33.3 \%$ (95\% CI; $27.7 \%$ - 40.6\%). The score on international HIV/AIDS dementia scale (IHDS) is as follows:

The first part on IHDS is timed finger tapping. On this part, motor speed is assessed, and $107(45.7 \%)$ did well, scoring 4 out of 4 . However, $34(14.5 \%), 17(7.3 \%)$ and $9(3.8 \%)$ did worse, scoring $2 / 4,1 / 4$ and $0 / 4$ respectively. On the next part, which assessed psychomotor speed, $125(53.4 \%)$ worked well without any impairment, scoring 4 out of 4 , also $19(8.1 \%)$, and $8(3.4 \%)$ scored one and zero out of 4 respectively. Memory recall was assessed in the third part, and it was found that $119(50.9 \%)$ recalled all the four things without any clue, scoring 4 out of 4 , but about $7(3 \%)$ could not recall all the things even with a clue, and they score zero out of 4 .

The magnitude across sex reveals that $34.4 \%$ of women and $31.3 \%$ of men developed NCD. Similarly, the problem increased with increment in age. Thus, it was $25 \%, 25.3 \%$ and $49.4 \%$ for the age groups of 18 - 30 years, 31 - 40 years and above 40 years respectively. Besides, it was $36.4 \%$ for unmarried participants. Concerning occupation, higher magnitude was observed for farmers and merchants with a proportion of 50\% and $48.4 \%$ respectively. In the level of income, $58.6 \%$ of participants who earned above 1500 Birr and $37.5 \%$ of those who earned 500 -100 Birr per

DOI: http://dx.doi.org/10.4314/ejhs.v27i1.9 
month had NCD while $36.4 \%$ of the participants who were on HAART had NCD, 39.4\% of those on follow-up for 5 years or more had NCD. From the sub-group of clinical stages, $74.2 \%$ of the participants in clinical stages of stage 3 and stage 4 developed NCD. Based on the CD4 count, it was found that $64 \%$ of them with 200 cells/dl or less had NCD; and $39.7 \%$ of the participants with a count of 201 - 350 cells/dl had NCD. Similarly, the magnitude of NCD among participants who had impairment in their activity of daily living, among those who developed anxiety and among those who had depression was $82.6 \%, 38.5 \%$ and $37.6 \%$ respectively.

Other independent factors: Activity of daily living was assessed using Katz Index of Independence in Activity of Daily Living, and we found that about 23(9.8\%) of the participants had impairment in the function of activity of daily living. The levels of anxiety and depression as measured by Hospital Anxiety and Depression scale showed that $55.6 \%$ and $67.1 \%$ of HIV positive people had anxiety and depression respectively.
Substance use assessment: Of the total participants, 32(13.7\%) had used substance in the last three months. Alcohol was the commonest substance used by 14(6\%) of them. Both Tobacco and Khat use was also reported by $6(2.6 \%)$. Of substance users, about $11(34.3 \%)$ had HAND as determined by IHDS.

Factors associated with neurocognitive deficit: Variables with p-value of 0.2 or less in the bivariate logistic regression were selected for multivariate analysis. The final degree of association as determined by adjusted odds ratio revealed that HIV-positive people who had impairment in their activity of daily living were 7 times more prone to develop NCD $(\mathrm{AOR}=7.19$ (95\% CI;1.73, 21.83)).

In a similar pattern, being in the $3^{\text {rd }}$ stage of HIV/AIDS was a risk for NCD compared to stage 1. Thus, participants who were in the $3^{\text {rd }}$ stage were 4 times at risk of developing NCD compared to subjects in stage $1(\mathrm{AOR}=4.2(95 \% \mathrm{CI} ; 1.19$, 14.44)). Nonetheless, other socio-demographic characteristics, clinical factors, depression and anxiety were not significantly associated with NCD (Table 3).

Table 3: Multivariate association between neuro cognitive deficit and other factors, ACSH, Mekelle, Ethiopia, June 2016.

\begin{tabular}{|c|c|c|c|c|c|}
\hline \multirow[t]{2}{*}{ Variables } & & \multicolumn{2}{|c|}{$\begin{array}{l}\text { Neurocognitive } \\
\text { deficit }\end{array}$} & \multirow[t]{2}{*}{ COR $(95 \% \mathrm{CI})$} & \multirow[t]{2}{*}{ AOR $(95 \% \mathrm{CI})$} \\
\hline & & Yes & No & & \\
\hline \multirow[t]{2}{*}{ Depression } & Yes & 59 & 98 & $1.84(.99,3.38)$ & $1.15(.49,2.69)$ \\
\hline & No & 19 & 58 & 1 & 1 \\
\hline \multirow{2}{*}{ Anxiety } & Yes & 50 & 80 & $1.69(.97,2.97)$ & $1.04(.48,2.24)$ \\
\hline & No & 28 & 76 & 1 & 1 \\
\hline \multirow{3}{*}{$\begin{array}{l}\text { Duration of Illness } \\
\text { (in year) }\end{array}$} & 1 or less & 8 & 21 & 1 & 1 \\
\hline & $2-4$ & 20 & 58 & $.9(.35,2.36)$ & $.84(.29,2.46)$ \\
\hline & 5 or more & 50 & 77 & $1.7(.70,4.14)$ & $.79(.38,1.65$ \\
\hline \multirow[t]{4}{*}{ Clinical stage } & Stage I & 33 & 106 & 1 & 1 \\
\hline & Stage II & 22 & 42 & $1.68(.88,3.21)$ & $1.0(.45,2.24)$ \\
\hline & Stage III & 19 & 6 & $10.17(3.75,21.58)$ & $4.2(1.19,14.44)^{*}$ \\
\hline & Stage IV & 4 & 2 & $6.42(1.13,16.67)$ & $.61(.04,8.83)$ \\
\hline \multirow{4}{*}{$\begin{array}{l}\text { CD4 Count } \\
\text { (Cells/dl) }\end{array}$} & 200 or less & 16 & 9 & $6.29(2.26,17.49)$ & $2.39(.69,8.29)$ \\
\hline & $201-350$ & 29 & 44 & $2.33(1.08,5.06)$ & $1.19(.47,3.04)$ \\
\hline & $351-500$ & 20 & 57 & $1.24(.56,2.76)$ & $.93(.39,2.24)$ \\
\hline & $>500$ & 13 & 46 & 1 & 1 \\
\hline \multirow{2}{*}{$\begin{array}{l}\text { Impairment in } \\
\text { activity of daily } \\
\text { living }\end{array}$} & Yes & 19 & 4 & $12.24(3.99,21.48)$ & $7.19(1.73,21.83)^{*}$ \\
\hline & No & 59 & 152 & 1 & 1 \\
\hline
\end{tabular}

Where $\mathrm{COR}=\mathrm{Crude}$ odds ratio, $\mathrm{CI}=\mathrm{Confidence}$ interval, $\mathrm{CI}=$ Confidence interval, *significantly associated

DOI: http://dx.doi.org/10.4314/ejhs.v27i1.9 


\section{DISCUSSION}

This study was undertaken to evaluate neurocognitive dysfunction in HIV patients using IHDS. In another study, among 234 patients, $78(33.3 \%)$ had HAND. The prevalence of neurocognitive deficit in our study was higher than expected. Since most of our subjects were receiving treatment with HAART, higher prevalence might be observed. Yet, it is important to note that the high prevalence of neurocognitive deficit in this study may reflect the need for further refinement of the tool, IHDS, at national level. As compared to prior studies, the current finding is similar with the result of a study conducted in India (32.5\%) (18), a study done in Nigeria (34.4\%) [28] and an earlier Metaanalysis (systemic review) in Sub-Saharan area (30.9\%) (2). However, it is less than the findings of previous studies undertaken in Botswana (38\%) (21), a study in the international community (37\%) (15), a study done in Cameroon (85\%) [29], a study conducted in Asia (90\%) (19) and a Ugandan study $(46.49 \%)(30,31,32)$.

Most of the participants among whom higher prevalence was reported might be in late stages of the illness by which neurocognitive impairment is more likely. Additionally, the other reason might be accounted to the difference in rating scales along with the variance in socio-demographic characteristics. However, the current finding is high compared to studies from Ethiopia (24.8\%) and another study from Nigeria $(21.5 \%)(17,22)$. An earlier study from Ethiopia that employed IHDS determined the more severe form of neurocognitive deficit (HIV associated dementia). That could be the reason for the discrepancy. Other socio-demographic variations might also play a role for the inconsistency.

This result is very high compared to multicentered international studies (that were conducted at Thailand, Zaire, Kenya, Brazil and Germany). the problem was in a range of $13 \%$ to $19 \%$. Also, this result is higher than the study in Malawi where prevalence of NCD was $15 \%(14,15,16$, 20).

Different viral clades and screening tools (or the questionnaire) used may account for the variation in the NCD as certain clades may be more or less neuropathogenic $(33,34)$. Relatively, neurocognitive deficit is more prevalent in regions where subtype C HIV predominates. As one study reported, $60 \%$ of subjects with subtype C-HIV were found to have NCD although they have no functional impairment clinically $(35,36)$.

The predominance of female participants in this study indicates that HIV/AIDS infection is more common in women than in men in Ethiopia. There are many reasons for this finding. These include cultural and economic factors that make women have little control over sexual and reproductive health issues. Another reason is that men do not come to the hospital either because of stigma or because they do not know their HIV status. Moreover, women are usually screened during antenatal visits as part of prevention of mother to child transmission of HIV. When found to be positive they are referred for treatment. Lastly, the finding of this study indicating that HIV infection is more common in women is similar with the observation made in Ethiopia. The result of this study showed that around $20.5 \%$ of the participants were unemployed, one-third of them had no education, and half of them had low level of monthly income which is similar with the country's profile (37).

Among those who had HIV associated neurocognitive deficit, one-fourth had depression as reported in a stud done in Botswana y (21). In our current study, as measured by HADS, more than half of the participants had depressive symptoms although it was not significantly associated with HAND.

Furthermore, participants classified with neurocognitive impairment using the IHDS were basically asymptomatic in our study, since $62(79.5 \%)$ out of 78 of those who had HAND did not report problems with daily activities or complain of cognitive problems. This might be alarming for clinicians and service providers to adopt and routinely screen HIV-positive people for possible NCD.

Late clinical stage was a predictor for the onset of NCD in this study. This is supported by prior studies from Ethiopia in which all of the subjects in late stages were found to have HIV associated dementia (more severe form of HAND). Also, in a Nigerian study, it was

DOI: http://dx.doi.org/10.4314/ejhs.v27i1.9 
deducted that as the disease progresses more and viral load is increased, the probability of HAND is likely (17). In Uganda where $31 \%$ of the subjects had HIV associated dementia, lower CD4 count and advanced HIV disease were risk factors for cognitive decline $(30,31,32)$. The study in Yaounde, Cameroon, which investigated risk factors for HIV associated neuricognitive deficit found that clinical factors of advanced clinical stage and lower CD4 count were predictors for HAND (38).

We found that NCD was significantly associated with impairment in the activity of daily living. Previous studies in India, Nigeria and Malawi found that symptomatic neurocognitive deficits were found in $2.5 \%, 9 \%$ and $12 \%$ of the participants respectively $(17,18,20)$. The more likely reason behind impaired daily functioning among HIV-positive people is NCD since it can impair motor activity besides memory and cognitive function $(4,11)$.

Different factors like older age, low level of education, lower level of hemoglobin, psychoactive substance use and opportunistic infections were mentioned as independent factors related to HIV associated NCD or HIV associated dementia in previous studies $(17,18,19,20,21,22)$. However, any of these factors did not show a significant association with NCD in our study.

In spite of the finding showing that $38.5 \%$ and $37.6 \%$ of the participants who had anxiety and depression had already developed NCD respectively, neither anxiety nor depression was significantly associated with NCD in this study.

We employed different standardized tools that help to determine the degree of association with the dependent variable. As a limitation, cause and effect relationship was not established between dependent and independent variables because of the cross sectional nature of the study.

The finding reveals that regardless of the use of HAART, HIV associated neurocognitive deficits were common problems especially among participants in late stages of the illness. In addition, it was significantly related with functional disability. Thus, it is highly crucial to prepare neurocognitive deficit screening tools at all clinical setups in order to recognize the problem early. Besides, it is vital for clinicians to routinely review the treatment regimen of patients and to monitor their clinical progress with special focus on those in late stages of the illness and those who are functionally disabled.

\section{ACKNOWLEDGMENT}

We are highly grateful to Ayder Comprehensive Specialized Hospital, Mekelle University, data collectors and study participants.

\section{REFERENCES}

1. UN/AIDS. Fact Sheets 2016. Available on www.unaids.org/resources/factsheets/

Accessed on 21 May, 2016.

2. Habib AG, Yakasai AM, Owolabi LF, Ibrahim A, Habib ZG, Gudaji M, et al. Neurocognitive impairment in HIV-1-infected adults in SubSaharan Africa: a systematic review and metaanalysis. International Journal of Infectious Diseases. 2013 Oct 31;17 (10):e820-31.

3. Saylor D, Dickens AM, Sackator N, Haughey $\mathrm{N}$, Slusher B, Pletnikov M, et al. HIV associated neurocognitive disorderpathogenesis and prospects for treatment. Nature Reviews Neurology. 2016; 12: 234-48. Doi: 10.1038/nrneurol.2016.27

4. Richard W Price. HIV Associated neurocognitive disorders: Epidemiology, clinical manifestations, and diagnosis. 2016. Up-to-date. Available at www.uptodate.com/cpntents-hiv-associatedneurocognitive-disorders-epedemiologyclinical-manifestations-and-diagnosis. Accessed on 10 Feb, 2016

5. Spudich SS. CROI 2014: neurologic complications of HIV infection. Top Antvir Med. 2014 May; 22 (2):594 - 601.

6. T Archer. Aspects of cognition/Health failure by HIV infected Individuals; Amelioration through exercise. HIV Curr Res. 2016; 1: e101

7. Woods SP, Moore DJ, Weber E, Grant I. Cognitive neuropsychology of HIV-associated neurocognitive disorders. Neuropsychology review. 2009 Jun 1;19(2):152-68.

8. Etherton MR, Lyons JL, Ard KL. HIVassociated neurocognitive disorders and antiretroviral therapy:current concepts and

DOI: http://dx.doi.org/10.4314/ejhs.v27i1.9 
controversies. Current infectious disease report. 2015 June; 17 (6):1- 6

9. Foca E, Agro P, Motta D, Compostella S, Casari S, Bonito A, et al. Screening for neuorcognitive impairment in HIV infected individuals at first contact after HIV diagnosis: the experience of a large clinical center in Northern Italy. International Journal of Molecular Sciences. 2016 Mar; 17 (4):434.

10. Catherine Adams, ShilpaZachari, Lisa Masters, Caroline Coffey and Pepe Catalan. Mental health problems in people living with HIV: changes in the last 2 decades: the London experience 1990-2014. AIDS Care. 2016; $28 \quad$ (1): 56-59. Doi: 10.1080/09540121.2016.1146211

11. Achappa B, Priyadarshni S, Madi D, Bhaskaran U, Ramapuram JT, Rao S, et al. Neurocognitive dysfunction among HIV positive patients using International HIV dementia scale. Asian Journal of Medical Sciences. 2014 May. 175(4):61-4.

12. Heaton RK, Clifford DB, Franklin DR Jr, Woods SP, Ake C, Vaida F, et al. HIVassociated neurocognitive disorders persist in the era of potent antiretroviral therapy: CHARTER Study. Neurology. 2010;75:20872096.

13. Anand P, Springer SA, Copenhaver MM and Altice FL. Neurocognitive Impairment and HIV Risk Factors: A Reciprocal Relationship. AIDS Behav. 2010;14:1213-1226.

14. Heaton RK, Clifford DB, Franklin DR Jr, Woods SP, Ake C, Vaida F, et al. Neuropsychological impairment in human immunodeficiency virus-infection: implications for employment. HNRC Group. HIV Neurobehavioral Research Center. Psychosom Med .1994 Jan-Feb;56(1):8-17

15. Robertson K, Liner J, Heaton R. Neuropsychological assessment of HIVinfected populations in international settings. Neuropsychol Rev. 2009, 19(2):232-249.

16. BanoitDube, Tami Benton, and Dwight L Evans. Neuropsychiatric Manifestations of HIV infection and AIDS. Canadian Medical Association. Journal of Psychiatry and Neuroscience. 20005 Sep; 30 (5):365.

17. AbdulkareemJika Yusuf, Abdulaziz Hassan,
Aisha Indo Mamman, Haruna Mohammed Muktar, et al. Prevalence of HIV Associated Neurocognitive Disorder (HAND) among Patients Attending a Teritary Health Facility in Northern Nigeria. Journal of the International Association of Providers of AIDS Care. Oct 2014. Doi: $10.1177 / 2325957414553839$

18. Savita Sain and Kivan B Barar. Assessment of Neurocognitive functions in HIV/AIDS patients on HAART using the International HIV Dementia Scale. International Journal of Nutrition, Pharmacology, Neurological Diseases. 2014; 4 (4):252-255.

19. Achappa B, Priyadarshni S, Madi D, Bhaskaran U, Ramapuram JT, Rao S, et al. Neurocognitive dysfunction among HIV positive patients using International HIV dementia scale. Asian Journal of Medical Sciences. 2014 May; 17;5(4):61-4.

20. Kelly CM, Van Oosterhout JJ, Ngwalo C, Stewart RC, Benjamin L, Robertson KR, et al. HIV Associated Neurocognitive Disorders (HAND) in Malawian Adults and Effect on Adherence to Combination Anti-Retroviral Therapy: A Cross Sectional Study. PLoS ONE. 2014; 9(6): e98962. doi:10.1371/journal.pone.0098962

21. Kathy L., Mosepele M., Sarah R., Esther S, Katherine S, Rudo N, et al. Neurocognitive impairment among HIV-positive individuals in Botswana: a pilot study. Journal of the international AIDS society. 2010, 13:15

22. Tilahun BM, Addisu WK, Minale TT. HIV Dementia among HIV Positive People at DebreMarkos Hospital, Northwest Ethiopia. American Journal of Psychiatry and Neuroscience. 2014; 2 (2):18-24.

23. Mekelle city Administration. About Mekelle. Available at: www.mekeleadministration.gov.et. Accessed on May 16, 2016

24. Sacktor N.C, Wong M, Nakasujja N, Skolasky R.L , Selnes O.A, Musis, S. et al. The International HIV Dementia Scale: a new rapid screening test for HIV dementia. AIDS. 2005; 19:1367-1374.

25. Khoei MA, Akbari ME and Sharifi F. Translation and validation of the Activities of 
Daily Living Scale with Iranian elderly cancer patients treated in an Oncology unit. Asia Pacific Journal of Cancer Prevention. 2013; 14 (4):3259.

26. Meredith Wallace. Katz Index of Independence in Activities of Daily Living (ADL). 2007; issue 2. Try this: General assessment series. Hartford Institute for Geriatric Nursing, New York University, College of Nursing

27. Reda AA. Reliability and validity of the Ethiopian version of the Hospital Anxiety and Depression Scale (HADS) in HIV infected patients. PLoS One. 2011; 6(1), e16049

28. Oshinake OO, AA Akinbami, OO Ojo, IF Ojini, UN Okubadejo and AM Danesi. Comparison of the Minimental State Examination Scale in Assessing Cognitive function in Nigerian HIV Patients on Aniretroviral Therapy. AIDS res Treat. 2012; 2012:581531.

29. Julius Atashili, Bradley N Gynes, Brian W Pence, Gladys T, Dimitry K, Julie K O'donnell, et al. Prevalence Characteristics and Correlates of a Positive Dementia Screen in Patients on Anteretroviral Therapy in Bamenda, Cameroon: A Cross-Sectional Study. BMC Neurology. 2013; 13:86.

30. Dore GJ. Marked improvement in survival following AIDS dementia complex in the era of HAART. AIDS. July 4, 2003; 17(10):153945

31. Sacktor N. The epidemiology of human immunodeficiency virus-associated neurological disease in the era of highly active antiretroviral therapy. Neurovirol. 2002; 8 (2):115-121.
32. McDermott MP, Sacktor N, Marder K, Schifitto G, Selnes OA, McArthur JC, et al. HIV-associated cognitive impairment before and after the advent of combination therapy. $J$ Neurovirol. 2002; 8:136-142.

33. Lovejoy TI, Suhr JA .The relationship between neuropsychological functioning and adherence in HIV-positive adults: a systematic review. J Behav Med.2009 Oct; 32(5):389405.

34. Tedaldi EM, Minniti NL, and Fischer T. HIV Associated Neurocognitive Disorders: The Relationship of HIV Infection with Physical and Social Comorbidities. Biomed Research International. 2015; doi 10.1155/215/641913

35. Haddow LJ, Floyd S, Copas A and Gilson RJ. A systematic review of screening accuracy of HIV Dementia Scale and International HIV dementia Scale. PLos One. 2013 Apr; 8(4): e61826.

36. de Almeida SM, Ribeiro CE, de Periera PE, Badiee J, Cherner M, Smith $\mathrm{D}$, et al. Neurocognitive impairment in HIV-1 clade-C versus B-infected individuals in Southern Brazil. J Neurovirol. 2013 Dec 2013; 19 (6): 550-6. doi: 10.1007/s13365-013-0215-5

37. HIV/AIDS Prevention and Control OfficeMinistry of Health, Ethiopia. 2016

Available at: www.moh.gov.et>hapco. Accessed on 10 May, 2016

38. Jamnshi A.K. Risk factors for HIV associated neurocognitive disorders in Sub-Saharan Africa: The case of Yaounde- Cameroon. Journal of the neurological sciences. October 15, 2009; 1-2(285):149-153. 